\title{
Reducción de la participación salarial en el ingreso y precarización del empleo
}

\author{
Daniel Velázquez Orihuela ${ }^{1}$
}

\begin{abstract}
"Manteniendo inseguro[s] a los trabajadores los tienes bajo control; de este modo no van a exigir salarios decentes ni condiciones laborales decentes, y tampoco el derecho

a la libre asociación, es decir, a formar sindicatos. Y a los dueños de la humanidad esto les va bien, ellos van sacando beneficios, pero para la población es devastador"
\end{abstract}

(Requiem for the American Dream, 2015)

\section{Resumen}

La globalización económica que se inició a finales de la década de 1970 y principios de la década de 1980 ha generado un estado de inseguridad laboral para los trabajadores. Este estado de inseguridad laboral coincide con dos tendencias: la reducción de la participación salarial en el ingreso y la precarización del empleo, ambas observadas en la mayoría de las economías de mercado desde principios de los años ochenta. El objetivo de este artículo es ofrecer una explicación sobre cómo la creciente inseguridad en el empleo ha provocado que la participación de los salarios en el ingreso se reduzca y que el empleo se precarice. Para cumplir con este objetivo se propone un modelo de salarios de eficiencia, en un escenario de equilibrio restringido por la demanda. Dicho modelo, además, tiene la virtud de explicar algunas características propias del mercado laboral de México en las últimas décadas.

\section{Palabras clave}

Empleo, mercado de trabajo, salarios, condiciones de empleo, relaciones laborales, ingresos, distribución del ingreso, modelos econométricos, México

\section{Clasificación JEL}

D33, E24, E25

\section{Autor}

Daniel Velázquez Orihuela es Profesor Investigador de tiempo completo del Área de Economía de la Universidad Autónoma del Estado de Hidalgo de México. Correo electrónico: daniel_velazquez7607@uaeh.edu.mx.

\footnotetext{
El autor agradece a los dictaminadores anónimos del artículo, a la maestra Mayra Vega Campa y a sus colegas del seminario permanente sobre la teoría de la inexistencia del mercado de trabajo por sus comentarios y sugerencias en relación con esta investigación.
} 


\section{Introducción}

La globalización productiva, que se inició a finales de los años setenta y principios de los ochenta, se ha caracterizado por la relocalización de la producción que llevan a cabo las empresas transnacionales para abaratar los costos laborales. Esta les ha permitido situar sus procesos intensivos en mano de obra en países donde existen bajos salarios y regulación laboral laxa. La amenaza de que las empresas emigren de sus países de origen ha provocado un estado de inseguridad en los trabajadores y ha reducido el poder de negociación de sus sindicatos (Kalleberg, 2009). Por otra parte, la caída del bloque socialista integró al mercado mundial a millones de personas; ante este exceso de fuerza de trabajo, muchos de los países pobres han optado por competir en la atracción de la inversión extranjera directa ofreciendo bajos salarios y regulaciones laborales flexibles o inexistentes que permiten y facilitan el libre despido, lo que ha generado una sensación de inseguridad en el empleo (Requiem for the American Dream, 2015). La sensación de inseguridad de los trabajadores respecto de la permanencia en el empleo es uno de los principales efectos que ha tenido la globalización productiva sobre los mercados de trabajo, y actualmente es una de las principales características en la mayoría de estos (Kalleberg, 2009).

La globalización productiva y la inseguridad en el empleo que generó coinciden con dos grandes tendencias: la reducción de la participación de la masa salarial en el ingreso y la precarización del empleo, que se iniciaron a la par que la globalización.

La caída de la participación de los salarios en el ingreso y el crecimiento de la precarización del empleo son dos tendencias que se han observado tanto en países ricos como en países pobres (FMI, 2007; OIT/OCDE, 2015; Kalleberg, 2009). Sin embargo, los países emergentes y en desarrollo han experimentado una reducción más pronunciada y acelerada de la participación de los salarios en el ingreso nacional (OIT, 2011; Stockhammer, 2009) y un crecimiento más rápido del trabajo precario (Vázquez, Macias y Lawson, 2018) que los observados en los países ricos.

De acuerdo con la OIT (2018), en los países emergentes el 46\% de los trabajadores tienen empleos vulnerables; esta cifra se eleva a un $76 \%$ en los países pobres. En la otra cara de este fenómeno, se observa que en Asia la participación del salario en el ingreso nacional ha caído más de 20 puntos porcentuales desde 1994, y en los países del norte de África, más de 30 puntos porcentuales desde el año 2000.

En América Latina la caída ha sido más moderada, pues apenas supera el 10\% desde 1993. Sin embargo, en esta región se observa una aceleración del descenso (OIT, 2011). Abeles, Amarante y Vega (2014) sostienen que la reducción de la participación del trabajo en el producto se da en la mayoría de los países latinoamericanos, aun si se consideran los ingresos de los trabajadores independientes. Alarco $(2014,2016$ y 2017) argumenta que en la mayoría de los países de América Latina se observa una historia común en que la participación salarial en el período 1950-2012 muestra dos ciclos completos: el primer máximo se sitúa a mediados de la década de 1960; el primer mínimo, a mediados de la década de 1980; el segundo máximo, a mediados de la década de 1990, y el segundo mínimo, entre 2004 y $2006^{2}$. No obstante, hay una tendencia descendente de la participación de la masa salarial: dicha participación fue más alta entre 1950 y 1979 que durante el lapso de 1980 a 2014, es decir, la máxima participación salarial se alcanzó cuando el modelo de industrialización por sustitución de importaciones era más intenso o cuando las coyunturas sociopolíticas favorecían a los trabajadores; en cambio, los valores mínimos se producen a partir de la crisis de la deuda de 1980, cuando estaban vigentes las políticas promercado.

El objetivo de este artículo es mostrar que la inseguridad en el empleo es una de las causas que propician tanto el crecimiento de la precarización del empleo como la disminución de la participación

\footnotetext{
2 Durante la primera mitad del primer decenio de este siglo se observó una mejora de la distribución personal, pese a la reducción de la participación salarial. Esto puede deberse a una subestimación de los ingresos de los percentiles más prósperos (Abeles, Amarante y Vega, 2014).
} 
de los salarios en el ingreso. Para ello se construye un modelo de salarios de eficiencia en un escenario de equilibrio restringido por la demanda. En dicho esquema analítico se estudian los mecanismos a través de los cuales el incremento de la inseguridad en el empleo ocasiona que los puestos de trabajo se precaricen y la participación de los salarios en el ingreso se reduzca. Además, el modelo tiene la virtud de reproducir y explicar algunas de las características propias del mercado de trabajo en México.

El artículo se divide en cinco secciones: tras la presente introducción, se enumeran en la segunda sección algunas de las características del mercado de trabajo mexicano. En la tercera sección se construye un modelo de salarios de eficiencia en un escenario de equilibrio restringido por la demanda para ofrecer una explicación común a la concentración del ingreso, a la precarización del trabajo y a las características del mercado de trabajo de México, expuestas en la segunda sección. En la cuarta sección se presenta un ejemplo numérico del modelo teórico. Finalmente, la quinta sección recoge las conclusiones del trabajo.

\section{Algunas características del mercado de trabajo en México}

En las últimas décadas la concentración del ingreso y la precarización del empleo han sido la regla en la economía mexicana, y se han presentado acompañadas de las siguientes tendencias:

i) La participación del ingreso laboral en la renta nacional venía reduciéndose desde 1976 hasta la actualidad. No obstante, en la última década la caída de la participación del ingreso laboral se ha acelerado (Ros, 2015; Samaniego, 2014; Luyano y Herrera, 2018).

ii) Desde 2008 se ha producido una convergencia salarial a la baja, y se observa que la mediana de los salarios altos ha caído más rápido que la de los bajos. Esto ha reducido la brecha salarial entre los distintos trabajadores, ya sea que se clasifiquen de acuerdo con su posición ocupacional, por años de escolaridad o por laborar en el sector formal o informal de la economía (Colmex, 2018).

En las últimas dos décadas se observa lo siguiente:

iii) El número de empleos mal remunerados ha crecido; en contraste, se han reducido los empleos bien remunerados ${ }^{3}$.

iv) Han aumentado los empleos precarios (entendidos como aquellos que carecen de acceso a seguridad social, no ofrecen contrato o bien ofrecen solo un contrato temporal y cuya remuneración es igual o menor que un salario mínimo) (Colmex, 2018).

v) La tasa de crecimiento de la productividad laboral media se ha reducido. En contraste, la productividad de la industria manufacturera de bienes comerciables ha crecido rápidamente (Ros, 2013 y 2015).

Con la finalidad de ofrecer una explicación de las tendencias descritas, se presenta a continuación un modelo de salarios de eficiencia en escenarios de equilibrio restringido por la demanda, haciendo especial hincapié en los procesos que concentran el ingreso y precarizan el empleo. Es importante aclarar que en el modelo se representa la precarización del empleo a partir de tres características: reducción del salario, incremento del esfuerzo que realizan los trabajadores y aumento de la inseguridad en el empleo ${ }^{4}$.

3 Como ejemplo de lo anterior: i) en 2008 había un millón y medio más de trabajadores que ganaban más de tres salarios mínimos que en 2014 y ii) el porcentaje de trabajadores subordinados y remunerados con ingresos laborales inferiores a un salario mínimo se incrementó de un 14,4\% en 2000 a un 24,0\% en 2017 (Samaniego, 2014; Colmex, 2018).

4 No existe un consenso sobre todas las características que debe incluir la categoría "trabajo precario". Sin embargo, en la mayoría de la literatura especializada, se incluyen como rasgos de esta categoría los bajos salarios, la inseguridad en el empleo y la intensidad o duración de la jornada laboral. 


\section{El modelo}

\section{Condiciones iniciales}

Se parte de una economía integrada por un gran número de productores y consumidores. Los consumidores se pueden clasificar en dos clases sociales. Sin embargo, todos los integrantes de cada clase tienen el mismo conjunto de gustos y preferencias y, de manera análoga, todas las empresas tienen la misma función de producción, por lo que es posible trabajar con una empresa representativa de todas las demás y un consumidor representativo de cada clase social.

Las clases sociales se distinguen entre sí por dos características: i) su fuente de ingresos y ii) sus gustos y preferencias. Distinguir a las clases sociales por su fuente de ingresos es una idea propia de la teoría clásica y marxista. Así, los trabajadores cuya única fuente de ingresos es su trabajo, utilizado y remunerado por las empresas, son llamados proletarios, y los dueños del capital, cuya principal fuente de ingresos son las ganancias de las empresas que poseen, son llamados capitalistas. Siguiendo esta tradición nombraremos a una clase social "proletariado" y a la otra "capitalista". Además de la diferencia basada en el origen de su ingreso, asumiremos que los capitalistas suelen demandar trabajo para incrementar su ocio o hacerlo más placentero.

En este modelo los proletarios tienen dos opciones para emplearse: la primera es en las empresas, la segunda es el autoempleo. A la primera de ellas se le llamará empleo convencional. Los trabajadores que se autoemplean no tienen el capital ni la organización para constituir una empresa formal, por lo que ofrecen su trabajo a los consumidores capitalistas con la finalidad de incrementar o hacer más placentero el ocio de estos. El único ingreso que reciben los autoempleados es el salario que les pagan los consumidores capitalistas. Esto implica que existen dos sectores productivos: un sector convencional, en el que las empresas producen bienes, y un sector en el que los autoempleados ofrecen sus servicios.

Existe información asimétrica e imperfecta, por lo que ni las empresas ni los consumidores capitalistas pueden identificar quién de sus trabajadores se esfuerza y quién no. Se asumirá que los trabajadores se esfuerzan a medida que aumentan los salarios o la probabilidad subjetiva de despido, por lo que todos los contratantes de trabajo pagan un salario de eficiencia, es decir, pagan el salario que maximice su tasa de ganancia o su utilidad, según corresponda.

La relación positiva entre los salarios y el esfuerzo y entre la probabilidad de despido y el esfuerzo son hipótesis estándares en la teoría del salario de eficiencia (Caraballo, 1996). La probabilidad de despido suele representarse por medio de la tasa de desempleo. Sin embargo, en este artículo se asume que la probabilidad subjetiva de despido refleja la sensación de inseguridad en el empleo que tienen los trabajadores, por lo que, además de la tasa de desempleo, se incorporan variables como la existencia y duración del contrato, la existencia de seguridad social, la flexibilidad de la legislación laboral y el respeto a los derechos laborales, entre otros aspectos ${ }^{5}$. En consecuencia, esta probabilidad puede incrementarse sin que la tasa de desempleo lo haga.

Los autoempleados tienen menos estabilidad en el empleo, es decir, pueden ser despedidos con mayor facilidad que los trabajadores empleados en las empresas. Esta idea se sustenta en que los autoempleados usualmente carecen de sindicatos, seguridad social, contratos escritos y protección legal. Al respecto, el Instituto Nacional de Estadística y Geografía de México (INEGI) muestra que, en la economía mexicana, menos del 1\% de los trabajadores autoempleados tienen algún tipo de seguridad social (INEGI, 2017).

\footnotetext{
5 De acuerdo con la Corporación Latinobarómetro (2005), el 75\% de los trabajadores en América Latina tenían miedo de perder su empleo en los siguientes 12 meses, y este porcentaje se elevó al 82\% en el caso de los trabajadores mexicanos.
} 
La probabilidad subjetiva de despido de los autoempleados $\left(p_{b}\right)$ depende de forma positiva de la probabilidad subjetiva de despido de los trabajadores convencionales $\left(p_{a}\right)$. Esta hipótesis se sustenta en que cuando el desempleo aumenta, los desempleados pueden optar por autoemplearse, incrementando así la oferta de servicios que brindan los autoempleados, haciendo más difícil la contratación para todos ellos. En esta lógica, Ros (2013) reconoce que el sector de los servicios de baja productividad se ha convertido en el último escaño por el que transitan los trabajadores mexicanos antes de caer en el desempleo.

Las ideas expresadas en los dos párrafos anteriores se formalizan en las siguientes hipótesis: $0<p_{a}<p_{b}<1$ y $0<\frac{d p_{b}}{d p_{a}}<1$.

\section{Empresa representativa}

La conducta optimizadora de la empresa es formalizada a partir del siguiente ejercicio de maximización:

Sujeto a

$$
\max (1+\pi)=\frac{y_{0}}{w l_{a}}
$$

En las expresiones (1) y (2), $(1+\pi)$ es la tasa de ganancia, $y_{0}$ es la oferta de producto, $w$ es el salario real, $l_{a}$ es la demanda de trabajo que realiza la empresa, $e\left(w, p_{a}\right)$ es la función de esfuerzo y $p_{a}$ es la probabilidad subjetiva de ser despedido por la empresa.

Donde $A \in \Re^{+}, e_{w}^{\prime}>0, e_{w}^{\prime \prime}<0, e_{p_{a}}^{\prime}>0, e_{p_{a}}^{\prime \prime}<0, e_{w, p_{a}}^{\prime \prime}=0$ y $e(w, 0)>0,0<p_{a}<1$ y $f$ es una función homogénea de grado 1.

De la formalización del axioma de racionalidad de la empresa se destacan dos características:

i) Las empresas tienen como objetivo la maximización de la tasa de ganancia, que se define como el cociente del valor de las ventas sobre el valor de los costos. Esta idea contrasta con la habitual de la teoría neoclásica, que postula que las empresas maximizan la ganancia (la diferencia entre el valor de las ventas y el de los costos). No obstante, es coherente con la idea clásica y marxista de que la principal variable que guía las decisiones de las empresas es la tasa de ganancia. Más recientemente, la teoría de la inversión financiera resalta las distintas tasas de rentabilidad como las variables claves que emplean los inversionistas para decidir dónde invertir.

ii) De manera análoga a lo que indicó Solow (1979), para la empresa individual el salario tiene dos características: i) forma parte de los costos laborales y ii) estimula a los trabajadores a esforzarse. En consecuencia, las empresas pagan el salario que les garantice el mayor esfuerzo del trabajador al menor costo posible, es decir, el salario que les permita obtener la mayor tasa de ganancia posible.

Resolviendo el ejercicio de maximización ${ }^{6}$ (ecuaciones (1) y (2)) se obtiene:

$$
\begin{gathered}
\frac{e_{w}^{\prime}}{e\left(w, p_{a}\right)} w=1 \\
A f_{l_{a}}^{\prime}=\frac{A f\left(e\left(w, p_{a}\right) l_{a}\right)}{l_{a}}
\end{gathered}
$$

6 Véase la sección 1 del anexo A1. 
La ecuación (3) es la ecuación de Solow y muestra que los productores obtienen la máxima ganancia cuando el salario que pagan garantiza que la elasticidad salario-esfuerzo sea igual a la unidad. Al salario que satisface esta condición para las empresas se le llama salario de eficiencia $\left(w^{a}\right)$.

La ecuación (4) es una condición que usualmente se obtiene en los esquemas analíticos que proponen la maximización de la tasa de ganancia. Esta muestra que las empresas maximizan cuando el producto medio iguala al producto marginal del trabajo (Noriega, 2001; Velázquez y Hernández, 2018; Velázquez, Vargas y Hernández, 2019).

Adviértase que la ecuación (3) implica que los productores fijan el salario independientemente de la oferta y la demanda de trabajo, por lo que nada garantiza que el salario de eficiencia sea de pleno empleo. Por otra parte, debido a que la función de producción es homogénea de grado 1, la ecuación (4) se verifica para toda demanda de trabajo positiva, y la tasa de ganancia óptima no depende del nivel de empleo, pero sí del salario:

$$
\max (1+\pi)=\frac{A e\left(w^{a}, p_{a}\right)}{w^{a}}
$$

A diferencia de la tasa de ganancia, la masa de ganancia sí depende del nivel de empleo. Siempre que las empresas ofrezcan el salario de eficiencia, la masa de ganancia será:

$$
\Pi=\left(A e\left(w^{a}, p_{a}\right)-w^{a}\right) l_{a}
$$

Adviértase que la condición de Solow implica que el salario de eficiencia es: $w^{a}=\frac{e\left(w^{a}, p_{a}\right)}{e_{w}^{\prime}}$. Por ello, siempre que $A>\frac{1}{e_{w}^{\prime}}$, la tasa de ganancia será positiva y la masa de ganancia crecerá a medida que crezca el empleo, de modo tal que: $\frac{d \Pi}{d l_{a}}=A e\left(w^{a}, p_{a}\right)-w^{a}>0, \frac{d^{2} \Pi}{d l_{a}^{2}}=0_{\mathrm{y}} \lim _{l_{a} \rightarrow \infty} \Pi=\infty$. Lo anterior no implica que no sea posible conocer el nivel de empleo o que haya sistemáticamente pleno empleo, debido a que las empresas producirán solo aquello que el mercado les demande, pues si producen más acumularán inventarios no deseados y con ello pérdidas. En consecuencia, las empresas ajustan su producción a la demanda efectiva vigente $\left(\hat{y}_{d}\right)$, y esto determina el máximo empleo a contratar. Sin embargo, como se mostrará en el apartado 5 de esta sección III, no existe un mecanismo de mercado que garantice sistemáticamente una demanda efectiva compatible con el pleno empleo.

Como consecuencia de lo expuesto en el párrafo anterior, se tiene:

$$
\begin{gathered}
\hat{y}_{d}=A e\left(w^{a}, p_{a}\right) l_{a} \\
l_{d}=\frac{\hat{y}_{d}}{A e\left(w^{a}, p_{a}\right)}
\end{gathered}
$$

La ecuación (7) simplemente muestra que la producción se ajusta a la demanda efectiva vigente como condición para obtener la máxima ganancia posible; la ecuación (8) expresa la demanda de trabajo de las empresas y es análoga a la función de ocupación propuesta por Keynes (1936) ya que, al igual que esta última, muestra que el nivel de empleo depende positivamente de la demanda efectiva. 


\section{Consumidor proletario}

La conducta racional del consumidor proletario se formaliza a partir del siguiente ejercicio de maximización:

$$
\max U^{p}=\alpha \ln c^{p}+(1-\alpha) \ln \left(\tau^{p}-e\left(w, p_{i}\right) l_{0}\right)
$$

Sujeto a

$$
w l_{0}=c^{p}
$$

En las expresiones (9) y (10), $p_{i}$ para todo $i=a, b$ es la probabilidad subjetiva de ser despedido si se trabaja en las empresas $(i=a)$ o si se es autoempleado $(i=b), 0<\alpha<1, c$ es la demanda de consumo, $\tau$ es el tiempo biológicamente disponible para trabajar y $l_{0}$ es la oferta de trabajo de un trabajador convencional o de un autoempleado, según corresponda. El superíndice $p$ hace referencia a que se trata del consumidor representativo de la clase proletaria. Se resaltan dos aspectos de la formalización de la conducta racional del consumidor proletario:

i) La función de utilidad es una función Cobb-Douglas. Por ello, es una función de utilidad bien comportada.

ii) Los consumidores proletarios ofertan trabajo eficiente $\left(e\left(w, p_{i}\right) l_{0}\right)$. Por lo tanto, un incremento de su esfuerzo, ceteris paribus, reducirá su utilidad.

A partir del ejercicio de maximización se obtienen la demanda de consumo y la oferta de trabajo:

$$
\begin{gathered}
c^{p}=\frac{\alpha \tau^{p} w}{e\left(w, p_{i}\right)} \\
l_{0}=\frac{\alpha \tau^{p}}{e\left(w, p_{i}\right)}
\end{gathered}
$$

Las ecuaciones (11) y (12) representan planes de compra y venta habituales. Resalta que la oferta de trabajo no es constante, sino que cambia conforme se modifica el esfuerzo del trabajador.

\section{Consumidor capitalista}

La conducta racional del consumidor representativo de la clase capitalista se formaliza a partir del siguiente ejercicio de maximización:

$$
\max U^{c}=\beta \ln c^{c}+(1-\beta) \ln \left(e\left(w, p_{b}\right) l_{b}\right)
$$

Sujeto a

$$
\Pi=c^{c}+w l_{b}
$$

En las expresiones (13) y (14) $0<\beta<1$, el superíndice $c$ hace referencia a que se trata del consumidor representativo de la clase capitalista y $l_{b}$ es la demanda de trabajo que realiza el consumidor capitalista.

En la formalización del axioma de racionalidad del consumidor capitalista se destacan cuatro características: 
i) De manera análoga a la función de utilidad del consumidor proletario, la función de utilidad del consumidor capitalista es una función Cobb-Douglas.

ii) La idea de que la única fuente de ingresos del consumidor capitalista es la ganancia que recibe de las empresas de las que es dueño implica que no oferta trabajo. Esta es la razón por la que la oferta de trabajo no aparece ni en su función de utilidad ni en su restricción presupuestaria.

iii) La hipótesis de que el consumidor capitalista demanda trabajo para incrementar o hacer más placentero su ocio se formaliza al introducir su demanda de trabajo multiplicada por el esfuerzo en su función de utilidad.

iv) El consumidor capitalista, al igual que la empresa, está consciente de que el esfuerzo de los trabajadores depende del salario que se les paga; por ello, oferta un salario que maximice el esfuerzo de los trabajadores y minimice el costo laboral.

Del ejercicio de maximización se obtienen las demandas del consumidor capitalista y el salario que garantiza su máxima utilidad:

$$
\begin{gathered}
l_{b}=(1-\beta) \frac{\Pi}{w} \\
c^{c}=\beta \Pi \\
\frac{e_{w}^{\prime}}{e\left(w, p_{b}\right)} w=1
\end{gathered}
$$

La ecuación (15) representa la demanda de trabajo del consumidor capitalista, es decir, la demanda de trabajo realizado por los autoempleados. Esta depende positivamente de la ganancia y negativamente del salario. La ecuación (16) representa la demanda de consumo y simplemente muestra que los capitalistas consumen una proporción de su ganancia. La ecuación (17) es la ecuación de Solow y muestra que los consumidores capitalistas maximizan su utilidad cuando pagan un salario que garantice que la elasticidad salario-esfuerzo sea igual a 1, es decir, cuando pagan salarios de eficiencia $\left(w^{b}\right)$. Sin embargo, el salario de eficiencia que pagan las empresas $\left(w^{a}\right)$ no tiene que ser necesariamente igual al salario que ofrecen los consumidores capitalistas $\left(w^{b}\right)$. Esto se debe a que los autoempleados y los trabajadores empleados por las empresas tienen distintas probabilidades subjetivas de ser despedidos.

Al comparar los salarios que ofrecen las empresas con los que reciben los autoempleados se tiene que: dado que $P_{a}<p_{b}$, entonces $w^{b}<w^{a}$; es decir, debido a que los autoempleados tienen una mayor probabilidad subjetiva de ser despedidos, su salario es menor que el ofertado por las empresas. La razón de esto es que la mayor sensación de inseguridad en el empleo que tienen los autoempleados los fuerza a esforzarse más que los trabajadores convencionales para evitar el desempleo; en consecuencia, los consumidores capitalistas pueden pagar salarios menores que las empresas y aun así obtener el mismo esfuerzo de sus trabajadores.

\section{Equilibrio general restringido por la demanda}

El equilibrio restringido por la demanda son los vectores de precios y asignaciones que se verifican cuando la producción se ajusta sistemáticamente a la demanda efectiva. Sin embargo, esta última no es lo suficientemente grande para garantizar el pleno empleo. En la literatura, el equilibrio restringido por la demanda surge usualmente debido a que los precios se ajustan con lentitud y, por tanto, 
los agentes realizan transacciones fuera del equilibrio (Argandoña, Gámez y Mochón, 1996). A diferencia de lo que señala dicha literatura, pero de manera análoga a lo expuesto por Velázquez y Hernández (2018), en este artículo se plantea que el equilibrio restringido por la demanda se debe a que los planes de compra de trabajo de las empresas no tienen una relación inversa con el salario real (véase la ecuación (8) y a que los salarios son independientes de la oferta y la demanda de trabajo (véanse las ecuaciones (3) y (17)).

Los mercados de bienes y de trabajo se representan mediante las siguientes ecuaciones:

$$
\begin{gathered}
\hat{y}_{d}-y_{0}=0 \\
l_{a}-\phi l_{0}=0 \\
l_{b}-(1-\phi) \gamma l_{0}=0
\end{gathered}
$$

La ecuación (18) representa el mercado de bienes; la demanda efectiva $\left(\hat{y}_{d}\right)$ es la suma de las demandas financiables, es decir, de los planes de consumo que los agentes pueden costear con las ganancias que reciben de las empresas o con la remuneración que obtienen al ofrecer su trabajo y ser empleados. La ecuación (19) representa el mercado de trabajo "convencional", que vincula la demanda de trabajo de las empresas con la oferta de los trabajadores; $\phi$ es la proporción de la oferta de trabajo que se emplea en este mercado, donde $\phi \in(0,1)$. La ecuación (20) representa el mercado de trabajo de los autoempleados, que relaciona la demanda de trabajo de los consumidores capitalistas con la oferta de trabajo de los autoempleados; $\gamma$ es el porcentaje de trabajo empleado en este mercado; en consecuencia, $(1-\gamma)(1-\phi)$ es la tasa de desempleo ${ }^{7}$. A partir de este momento, llamaremos a la ecuación (19) "mercado de trabajo convencional" y a la ecuación (20) "mercado de trabajo del autoempleo".

Los salarios en los mercados de trabajo convencional y del autoempleo están determinados por las ecuaciones (3) y (17), respectivamente. A partir de este momento asumiremos que los salarios que se pagan en estos mercados son los de eficiencia.

La demanda efectiva está integrada por la suma de las demandas financiables del consumidor capitalista y del proletario. Considerando las ecuaciones (11) y (16), se tiene:

$$
\hat{y}_{d}=\beta \Pi+\phi \frac{\alpha \tau^{p} w^{a}}{e\left(w^{a}, p_{a}\right)}+(1-\phi) \frac{\alpha \tau^{p} w^{b}}{e\left(w^{b}, p_{b}\right)} \gamma
$$

El primer término del lado derecho de la ecuación (21) es la demanda financiable del consumidor capitalista; el segundo y el tercero son la demanda que el consumidor proletario puede costear con la oferta de trabajo que logra que se emplee y se remunere en los mercados de trabajo convencional y del autoempleo, respectivamente. Al resolver el equilibrio en el mercado de bienes, se obtiene:

$$
A e\left(w^{a}, p_{a}\right) l_{a}-\phi \frac{\alpha \tau^{p} w^{a}}{e\left(w^{a}, p_{a}\right)}+\phi \frac{\alpha \tau^{p} w^{a}}{e\left(w^{a}, p_{a}\right)}-A e\left(w^{a}, p_{a}\right) l_{a}=0
$$

\footnotetext{
7 La oferta de trabajo contratada por las empresas es $\phi l_{0}$ y la oferta de trabajo contratada por los consumidores capitalistas es $(1-\phi) \gamma l_{0}$. En consecuencia, la oferta de trabajo desempleada es $(1-\phi)(1-\gamma) l_{0}$ y la tasa de desempleo es $(1-\phi)(1-\gamma)$.

8 Véase la sección 2 del anexo A1.
} 
La ecuación (22) muestra que el equilibrio restringido por la demanda en el mercado de bienes se garantiza independientemente de los valores de $\phi$ y $\gamma$. Es decir, cualquiera que sea la distribución del empleo entre los mercados y cualquiera que sea la tasa de desempleo, la producción se ajustará a la demanda efectiva.

Adviértase que no es posible determinar el valor de $\phi$ sin asumir que se conoce la demanda efectiva (ecuación (8)) o el nivel de empleo que ocupan las empresas. No obstante, si se asume como conocido el valor de $\phi$ es posible determinar $\gamma$, que es:

$$
\gamma=\frac{\phi(1-\beta)}{(1-\phi)} \frac{w^{a}}{e\left(w^{b}, p_{b}\right)}\left(1-\frac{w^{a}}{e\left(w^{a}, p_{a}\right)}\right)
$$

La expresión (23) hace evidente que no existen mecanismos de mercado que garanticen que $\gamma$ sea igual a 1, es decir, que se verifique el pleno empleo. La razón de esto es que nada asegura que la demanda efectiva sea la adecuada para que haya pleno empleo. Este resultado difiere del obtenido habitualmente en los modelos de salarios de eficiencia; por ejemplo, en Shapiro y Stiglitz (1984) el equilibrio con desempleo se debe a que este último es necesario para garantizar el esfuerzo positivo de los trabajadores. En contraste, en este enfoque analítico el esfuerzo es positivo, pese a que la tasa subjetiva de despido sea 0.

\section{Condiciones iniciales para el análisis de la precarización del empleo y de la distribución del ingreso}

La precarización del empleo es un proceso dinámico que implica una caída de los salarios, una mayor inseguridad en cuanto a estar empleado y un incremento del esfuerzo que realizan los trabajadores. Para analizar la forma como una mayor precarización del empleo conlleva una mayor concentración del ingreso, se partirá de un equilibrio restringido por la demanda y se introducirá la hipótesis de decisión dual de Clower (1965), según la cual todos los agentes son compradores y vendedores, por lo que para comprar antes tuvieron que haber vendido. Lo anterior implica que la demanda efectiva depende del trabajo que el consumidor proletario logró vender y de las ganancias que las empresas obtuvieron por sus ventas, en ambos casos en el período pasado. Al incorporar esta hipótesis, se obtiene ${ }^{9}$ :

$$
\hat{y}_{d}=l_{a-1} w^{a}+\Pi_{-1}
$$

En la ecuación (24) el subíndice -1 hace referencia a que se trata de una variable del período pasado, por lo que podemos considerarla fija. La demanda efectiva expresada en la ecuación (24) tiene dos características: i) el salario no tiene el subíndice mencionado debido a que es una variable del período actual. La razón es que los trabajadores laboran durante un período (semana, quincena) y al final de este reciben su salario y posteriormente con este ingreso financian su consumo del siguiente período; ii) el consumo de los autoempleados no aparece como parte de la demanda efectiva. Esto se debe a que los capitalistas financian tanto su demanda de bienes como de trabajo con las ganancias que reciben de las empresas; en consecuencia, las ganancias incorporan tanto la demanda de consumo de los trabajadores que se autoemplean como la de los capitalistas.

9 Véase la sección 2 del anexo A1. 


\section{Precarización del empleo}

Para analizar la precarización del empleo se asume, como punto de partida, que la inseguridad en los empleos convencionales aumenta; por tanto, la probabilidad subjetiva de despido de los trabajadores que se emplean en las empresas se incrementa. En consecuencia, los trabajadores convencionales se esfuerzan más para evitar ser sorprendidos holgazaneando y, por ello, ser despedidos. El incremento del esfuerzo motiva a las empresas a reducir los salarios, debido a que ya no se requieren salarios altos para incentivar el esfuerzo. Así, sobre la base de la ecuación (3) se obtiene:

$$
\frac{d w^{a}}{d p_{a}}=\frac{e_{p_{a}}^{\prime}}{e_{w}^{\prime \prime} w^{a}}<0
$$

El aumento de la inseguridad en el empleo convencional incrementa la inseguridad en el autoempleo, debido a que, como ya se mencionó, los trabajadores que pierdan su empleo en las empresas buscarán autoemplearse, haciendo más difícil la contratación para todos los autoempleados. Así, $0<\frac{d p_{b}}{d p_{a}}<1$.

De forma análoga a lo que ocurre con los trabajadores convencionales, el incremento de la probabilidad subjetiva de despido de los autoempleados provoca que estos se esfuercen más para evitar el desempleo. El mayor esfuerzo motiva a los consumidores capitalistas a pagar menores salarios, pues sus trabajadores ya se están esforzando. Así, sobre la base de la ecuación (17) se obtiene:

$$
\frac{d w^{b}}{d p_{a}}=\frac{e_{p_{b}}^{\prime}}{e_{w}^{\prime \prime} w^{b}} \frac{d p_{b}}{d p_{a}}<0
$$

Las expresiones (25) y (26) muestran que los salarios tanto de los trabajadores empleados en las empresas como de los autoempleados disminuyen. La caída de los salarios se traduce en una reducción de la demanda efectiva. A partir de la ecuación (24) se tiene:

$$
\frac{d \hat{y}_{d}}{d p_{a}}=l_{1-1} w_{p_{a}}^{a}<0
$$

La ecuación (27) muestra que la demanda efectiva se reduce debido a que disminuye el poder de compra de los trabajadores que laboran en las empresas. Sin embargo, el menor ingreso de los autoempleados no afecta la demanda efectiva. Esto se debe a que una reducción del consumo de los trabajadores autoempleados será compensada por un aumento del consumo de los capitalistas, debido a que los consumidores capitalistas utilizan sus ganancias para contratar a los trabajadores que laboran para ellos, por lo que los consumos de ambos consumidores se financian en última instancia con las ganancias.

La caída de la demanda efectiva obliga a las empresas a ajustar su producción a la baja; por ello, requieren contratar a menos personas. A partir de la ecuación (8) se obtiene:

$$
\frac{d l_{a}}{d p_{a}}=\frac{1}{A}\left[\frac{\hat{y}_{d, p_{a}}^{\prime} e\left(w^{a}, p_{a}\right)-\left(e_{w^{a}}^{\prime} w_{p_{a}}^{a,}+e_{p_{a}}^{\prime}\right) \hat{y}_{d}}{e\left(w^{a}, p_{a}\right)^{2}}\right]<0
$$

La inecuación (28) muestra que hay dos razones por las cuales la demanda de trabajo de las empresas disminuye: i) el aumento del esfuerzo de los trabajadores $\left(e_{w^{a}}^{\prime} w_{p_{a}}^{a,}+p_{a} e_{p_{a}}^{\prime}\right)$ y ii) la disminución de la demanda efectiva $\left(\hat{y}_{d, p_{a}}^{\prime}\right)$. Existen dos características que resaltar de estos resultados: 
i) El cambio en el esfuerzo de los trabajadores obedece a dos efectos contrarios. Por un lado, la mayor inseguridad en el empleo motiva a los trabajadores a esforzarse más para no quedar desempleados; por el otro, el mayor esfuerzo que realizan permite a las empresas pagar menores salarios, lo que desmotiva a los trabajadores, provocando que reduzcan su esfuerzo. Por lo anterior, no es posible determinar a priori qué fuerza dominará. En este trabajo se postula que ante un incremento de la probabilidad subjetiva de despido los trabajadores incrementan su esfuerzo, pese a la reducción de los salarios. Lo anterior es cierto siempre que $\left.e_{p_{a}}^{\prime}\right\rangle\left|e_{w^{a}}^{\prime} w_{p_{a}}^{a}\right|$, lo que se verifica con la condición de que: $\left|e_{w}^{\prime \prime} w^{a}\right|>\left|e_{w}^{\prime}\right|$.

ii) La razón por la que una menor demanda efectiva implica una caída del nivel de empleo es que las empresas ajustan su producción a la baja para hacerla coincidir con la menor demanda efectiva, por lo que requieren menos trabajo.

Debido a que los empresarios ajustan su producción a la demanda efectiva vigente, se obtiene, sobre la base de la ecuación (18):

$$
\frac{d y_{0}}{d p_{a}}=\frac{d \hat{y}_{d}}{d p_{a}}
$$

La ecuación (29) muestra que la producción se reduce en el mismo monto que la demanda efectiva. La disminución tanto de la producción como de los costos laborales y el aumento del esfuerzo modifican las ganancias. Para analizar cómo cambian estas, se obtiene su deferencial con respecto a la probabilidad subjetiva de despido:

$$
\frac{d \Pi}{d p_{a}}=\left[A\left(e_{w^{a}}^{\prime} w_{p_{a}}^{a,}+e_{p_{a}}^{\prime}\right)-w_{p_{a}}^{a}\right] l_{a}+\left[A e\left(w^{a}, p_{a}\right)-w^{a}\right] l_{a, p_{a}}^{\prime}>0
$$

La ecuación (30) indica que la caída de los costos laborales y el incremento del esfuerzo de los trabajadores redundan en que la ganancia aumente, pese a que el menor nivel de empleo reduce la producción y, con ello, presiona la ganancia a la baja ${ }^{10}$. Es decir, la ganancia aumenta debido al crecimiento de la brecha entre la productividad del trabajo y el salario, no obstante la caída de las ventas. Por ello, en este escenario, el incremento de la ganancia implica un cambio en la distribución factorial del ingreso a favor de los capitalistas.

El incremento de la ganancia les permite a los consumidores capitalistas demandar más bienes de consumo y servicios ofertados por los autoempleados. Sobre la base de las ecuaciones (15) y (16) se obtiene:

$$
\begin{gathered}
\frac{d l_{b}}{d p_{a}}=(1-\beta) \frac{\Pi^{\prime}}{w^{b}}-(1-\beta) \frac{\Pi}{w^{b^{2}}} w_{p_{a}}^{b,}>0 \\
\frac{d c^{c}}{d p_{a}}=\beta \Pi^{\prime}>0
\end{gathered}
$$

La expresión (31) muestra que los consumidores capitalistas demandan más trabajo debido a que el precio de este se ha reducido y el ingreso de los capitalistas ha aumentado. En consecuencia, el empleo de los trabajadores autoempleados crece. Por otra parte, la inecuación (32) muestra que los capitalistas consumen más bienes debido al incremento de sus ingresos. Adviértase que la caída de la producción y el crecimiento del consumo de los capitalistas implican que el consumo de los proletarios

10 Véase la sección 3 del anexo A1. 
se reduce, pese a que aumentó el autoempleo; en contraste, el consumo de los capitalistas tanto de bienes como de servicios se incrementa. Lo anterior es una consecuencia del crecimiento de las ganancias, de la disminución de los salarios que reciben tanto los trabajadores convencionales como los autoempleados y de la reducción del empleo convencional.

\section{Análisis de algunas características del mercado laboral en México}

\section{a) Concentración del ingreso}

La primera característica que analizar es la disminución de la participación de los trabajadores en el ingreso nacional. En este modelo el ingreso nacional está integrado por el valor de la producción $\left(y_{0}\right)$ más el valor de los servicios $\left(w^{b} l_{b}\right)$ que proporcionan los trabajadores a los consumidores capitalistas. En consecuencia, la participación de las ganancias y de los salarios en el ingreso nacional (IN) está definida, respectivamente, por:

$$
\begin{gathered}
\frac{\Pi}{I N}=\frac{\Pi}{y_{0}+w^{b} l_{b}} \\
\frac{w^{a} l_{a}+w^{b} l_{b}}{I N}=1-\frac{\Pi}{y_{0}+w^{b} l_{b}}
\end{gathered}
$$

Adviértase que la ecuación (15) implica que $w^{b} l_{b}=(1-\beta) \Pi$, por lo que se obtiene:

$$
\frac{d \frac{\Pi}{I N}}{d p_{a}}=\frac{\Pi_{p_{a}}^{\prime}\left(y_{0}+(1-\beta) \Pi\right)-\Pi\left(y_{0, p_{a}}^{\prime}+(1-\beta) \Pi_{p_{a}}^{\prime}\right)}{\left(y_{0}+w^{b} l_{b}\right)^{2}}>0
$$

La ecuación (35) muestra que la participación de las ganancias en el ingreso nacional aumenta, por lo que la participación de los salarios se reduce. Lo anterior se debe a que el crecimiento de las ganancias es superior al incremento del ingreso nacional, debido a que la caída de la producción no es compensada por el aumento de los servicios en una medida suficiente para permitir que el ingreso nacional crezca por encima de las ganancias.

La concentración del ingreso en favor de las ganancias implica que la participación de los salarios en el ingreso disminuye, debido a la caída de los salarios, el crecimiento del empleo más precario (autoempleo) y la reducción del empleo convencional.

\section{b) Convergencia salarial a la baja}

La segunda característica que analizar es la convergencia salarial a la baja. De acuerdo con las ecuaciones (25) y (26), los salarios tanto de los trabajadores convencionales como de los autoempleados disminuyeron; sin embargo, para que la convergencia salarial a la baja se verifique es necesario que los salarios de los trabajadores convencionales se reduzcan en mayor medida que los de los autoempleados. Esto solo ocurre si $\frac{w^{b}}{w^{a}}>\frac{d p_{b}}{d p_{a}}$ o, expresado de otro modo, si $\frac{d p_{a}}{d p_{b}}>\frac{w^{a}}{w^{b}}$; es decir, si la diferencia entre el incremento de la inseguridad en el empleo convencional y en el autoempleo es superior a la brecha salarial. 


\section{c) Crecimiento del trabajo precario y disminución del empleo bien remunerado}

Las características tercera y cuarta del mercado de trabajo mexicano que interesa analizar son que el empleo precario aumenta, mientras que el empleo no precario se reduce. En términos del modelo aquí presentado, lo anterior implica que el empleo con remuneración más baja, más inseguro y que exige un mayor esfuerzo, es decir, el autoempleo, creció; en contraste, el empleo convencional disminuyó, lo que, de acuerdo con las inecuaciones (28) y (31), ocurre.

El empleo convencional disminuye debido a que la demanda efectiva que satisfacen las empresas se reduce a consecuencia de los menores salarios que reciben los trabajadores convencionales; en cambio, el autoempleo aumenta ya que el incremento de las ganancias y la reducción de los salarios de los autoempleados les permiten a los capitalistas demandar más servicios.

\section{d) La productividad manufacturera crece más rápido que la productividad media}

La última característica del mercado de trabajo de México que se analiza es el hecho de que la productividad media de bienes manufactureros comerciables crece más rápido que la productividad media total. Lo anterior implica que hay un sector productivo cuya productividad crece por encima de la media. Para analizar este fenómeno adviértase que por definición la productividad media del sector convencional es: $\frac{y_{0}}{l_{a}}=\frac{e\left(w^{a}, p_{a}\right) A l_{a}}{l_{a}}$. Por otra parte, la productividad media de los servicios ofrecidos por los autoempleados se define como: $\frac{w^{b} l_{b}}{l_{b}}$. Obsérvese que ninguna de las dos productividades depende del nivel de empleo, debido a que las dos son homogéneas de grado 0 en el empleo. Sin embargo, responden de forma opuesta al incremento de la inseguridad en el empleo.

La productividad en el sector convencional se incrementa ante el aumento de la probabilidad subjetiva de despido. Esto se debe a que si se incrementa la probabilidad subjetiva de despido, los trabajadores se esfuerzan más y, por tanto, son más productivos. Lo anterior se observa en la siguiente ecuación:

$$
\frac{d \frac{y_{0}}{l_{a}}}{d p_{a}}=A\left[e_{p_{a}}^{\prime}+e_{w}^{\prime} w_{p_{a}}^{a^{\prime}}\right]>0
$$

En contraste, la productividad media de los servicios ofertados por los autoempleados se reduce ante la mayor inseguridad en el empleo. La razón es que, de forma análoga a lo que ocurre con los trabajadores convencionales, si se incrementa la probabilidad subjetiva de despido, los trabajadores autoempleados se esfuerzan más; en consecuencia, los consumidores capitalistas pueden pagar salarios más bajos. Los menores salarios reducen el valor de los servicios ofertados, con lo que la productividad media del sector disminuye, de modo que:

$$
\frac{d \frac{w^{b} l_{b}}{l_{b}}}{d p_{a}}=w_{p_{b}}^{b^{\prime}} p_{b, p_{a}}^{\prime}<0
$$

La reducción de la productividad media de los servicios implica que la productividad media de la economía crece en menor medida que la productividad media del sector convencional, lo que concuerda con la información disponible sobre México. 
Los resultados presentados hasta aquí contrastan con la teoría poskaleckiana ${ }^{11}$, que estudia la distribución factorial del ingreso y sus efectos en el empleo y la producción. Entre las principales diferencias y similitudes resaltan:

i) En estos modelos poskaleckianos la reducción de la participación de los salarios en el producto se debe al incremento del sobreprecio (mark-up) y el grado de monopolio, que puede ser causado por depreciaciones del tipo de cambio (Bhaduri y Marglin, 1990), incrementos de la tasa de interés (Hein, 2014) o reducciones exógenas de los salarios reales. A diferencia de estos modelos, en el enfoque analítico aquí presentado, la caída de la participación de los salarios en el ingreso se produce sin asumir la existencia de monopolios en el mercado de bienes y, por tanto, sin recurrir a la hipótesis del sobreprecio. No obstante, postular salarios de eficiencia implica asumir mercados de trabajos no competitivos, en los que las empresas y los consumidores capitalistas puedan fijar los salarios de acuerdo con sus intereses. La razón es que los proletarios tienen que trabajar para vivir. En consecuencia, un incremento de la inseguridad en el empleo aumenta el poder de los contratantes de trabajo para fijar los salarios, lo que ocasiona la caída de la participación salarial.

ii) En estos modelos existen escenarios en que la producción es comandada por salarios o por ganancias. Estos resultados se basan en el reconocimiento del carácter dual de los salarios: como componentes de la demanda y como costos de la producción. De manera análoga a estos modelos, en este enfoque analítico se reconoce el carácter dual de los salarios; sin embargo, no existen mecanismos que permitan que la mayor rentabilidad de las empresas, sustentada en una reducción de los salarios, implique un crecimiento de la demanda efectiva, debido a que no se estudian la inversión ni las exportaciones netas, dos temas que son parte de la agenda pendiente de esta investigación.

\section{Ejercicio numérico}

Con la finalidad de ofrecer mayor claridad sobre el modelo teórico presentado, se expondrá un ejemplo numérico de este. Para ello se requiere una función de esfuerzo paramétricamente definida. Se asume una función de esfuerzo análoga a la propuesta por Velázquez y Hernández (2018), $e\left(w, p_{i}\right)=1-\frac{a}{w}+p_{i}^{\delta}$, donde $a \in \mathfrak{R}^{+}, 0<\delta<1$ y $0<p_{i}<1$ para todo $i=a, b$. Las características de esta función son ${ }^{12}$ :

i) $\quad e_{w}^{\prime}>0 ; e_{w}^{\prime \prime}<0 ; e_{p_{i}}^{\prime}>0 ; e_{p_{i}}^{\prime \prime}<0$ y $e_{w, p_{i}}^{\prime \prime}=0$

ii) $\quad e(w, 0)=1-\frac{a}{w}$

iii) $\quad e(w, 1)=2-\frac{a}{w}$

iv) $\quad \lim _{w \rightarrow 0} e\left(w, p_{i}\right)=-\infty$

v) $\quad \lim _{w \rightarrow \infty} e\left(w, p_{i}\right)=1+p_{i}^{\delta}$

Entre las características de la función de esfuerzo se destacan: i) cualquiera que sea la probabilidad subjetiva de despido, el esfuerzo será positivo. Sin embargo, a medida que los agentes esperen que esta crezca, incrementarán su esfuerzo; ii) si el salario tiende a 0, los trabajadores dejarán de esforzarse. Sin embargo, para salarios infinitos el esfuerzo está acotado.

\footnotetext{
${ }^{11}$ No es nuestro objetivo hacer una revisión exhaustiva de esta literatura. Véanse mayores detalles sobre ella en Hein (2014).

12 La principal diferencia entre la función propuesta por Velázquez y Hernández (2018) y la propuesta en este artículo radica en que en dicho trabajo los autores asumen que la probabilidad subjetiva de despido puede ser representada mediante la expectativa de desempleo.
} 
La relación entre la tasa subjetiva de despido de los autoempleados y la propia de los trabajadores convencionales está determinada por:

$$
p_{b}=\zeta+\varphi p_{a}
$$

Donde $0<\zeta<1 ; 0<\varphi<1$ y $0<p_{b}<p_{a}$.

La ecuación (38) muestra que la tasa subjetiva de despido de los autoempleados tiene una parte que no depende de lo que ocurra en el sector convencional $(\xi)$ y otra que transmite la inseguridad del sector convencional a este sector $(\varphi)$.

La función de producción será $y_{0}=A e\left(w, p_{a}\right) l_{a}$. Las funciones de utilidad serán las propuestas en las ecuaciones (8) y (12). Los valores numéricos de los parámetros de las funciones se especifican en el cuadro 1.

\section{Cuadro 1}

Parámetros de las funciones de producción, esfuerzo, probabilidad subjetiva de despido de los autoempleados y utilidad

\begin{tabular}{ccccccccc}
\hline $\mathrm{A}$ & $\mathrm{a}$ & $\delta$ & $p_{a}$ & $\zeta$ & $\varphi$ & $\alpha$ & $\beta$ & $\tau^{p}$ \\
10 & 1 & 0,9 & 0,01 & 0,05 & 0,9 & 0,4 & 0,9 & 99 \\
\hline
\end{tabular}

Fuente: Elaboración propia.

Nota: $\quad$ A a y $\delta$ son parámetros, el primero de la función de producción, los dos siguientes de la función de esfuerzo. $\alpha$ y $\beta$ son las elasticidades consumo-utilidad, la primera del consumidor proletario, la segunda del consumidor capitalista (véanse las funciones de producción, esfuerzo y utilidad).

El ejercicio numérico se expone en dos escenarios. En el primero de ellos se calcula un equilibrio restringido por la demanda. Para ello se supondrá conocido y dado exógenamente el porcentaje de la oferta de trabajo que se emplea en las empresas $(\phi)$; sin embargo, se prescinde de esta hipótesis en el escenario siguiente. Este primer escenario servirá como punto de partida para analizar la precarización del empleo y la concentración del ingreso. En el segundo escenario se asume que la probabilidad subjetiva de despido de los trabajadores convencionales aumenta; esto precariza el empleo, concentra el ingreso en favor de los dueños de las empresas, reduce la brecha salarial a la baja, incrementa el empleo precario, disminuye el empleo convencional e incrementa la productividad del sector convencional en mayor medida que la productividad media.

\section{Escenario 1: equilibrio restringido por la demanda}

Los parámetros utilizados en este escenario son los mismos que se emplearán en el escenario 2 (véase el cuadro 1).

De los parámetros elegidos resaltan las siguientes características: la probabilidad subjetiva de despido de los trabajadores convencionales $\left(p_{a}\right)$ es del 1\%; es decir, los trabajadores se sienten relativamente seguros en sus empleos. Se elige este valor para que en el siguiente escenario pueda crecer. Los proletarios son el $99 \%$ de la población $\left(\tau^{p}\right)$. A medida que la probabilidad subjetiva de despido de los trabajadores convencionales aumenta en 1,0 unidad, la de los autoempleados lo hace en 0,9; es decir, la transferencia de inseguridad en el empleo de un sector a otro $(\varphi)$ es alta. Suponemos esto debido a que, a falta de seguro de desempleo, los trabajadores desempleados en el sector convencional buscarán autoemplearse como último recurso ante la posibilidad del desempleo. Además de estos parámetros, se asume que el empleo formal representa el $80 \%$ de la oferta de trabajo, por lo que $\phi=0,8$. Este dato es congruente con lo que se señala en INEGI (2017) en el sentido de que los autoempleados en México representan cerca del 20\% del empleo total. Sobre la base de estos parámetros se obtienen las asignaciones que se presentan en el cuadro 2. 


\section{Cuadro 2}

Salarios, asignaciones y distribución en equilibrio restringido por la demanda

\begin{tabular}{cccccccc}
\hline$p_{a}$ & $p_{b}$ & $w^{a}$ & $w^{b}$ & $e\left(w^{a}, p_{a}\right)$ & $e\left(w^{b}, p_{b}\right)$ & $I_{a}$ & $I_{b}$ \\
\hline 0,01 & 0,059 & 1,968 & 1,854 & 0,507 & 0,539 & 62,371 & 10,459 \\
\hline$I_{b} \%$ & $u$ & $y_{0}$ & $S_{a}$ & $I N$ & $c^{c}$ & $c^{p}$ & $\% c_{t}^{c}$ \\
\hline 14,361 & 5,489 & 316,8 & 19,4 & 336,2 & 174,602 & 142,197 & 57,704 \\
\hline$\% c_{t}^{p}$ & $\Pi \sigma$ & $M . S$ & $\%$ & $\% M . S$ & $P m e s$ & $P m e c$ & $P m e t$ \\
\hline 42,295 & 194,003 & 142,197 & 57,704 & 42,295 & 1,854 & 5,079 & 4,616 \\
\hline
\end{tabular}

Fuente: Elaboración propia. respectivamente; el subíndice o superíndice "a" hace referencia al trabajador convencional y "b" al autoempleado; $I_{b} \%$ es el porcentaje que representan los autoempleados en el empleo total; $u$ es la tasa de desempleo; $y_{0}$ es la producción manufacturara; $S_{a}$ es el valor de los servicios; $I N$ es el ingreso nacional; $c^{c}, \% c^{c}, c^{p}$ y $\% c^{p}$ son el consumo de los capitalistas, su porcentaje respecto del consumo total, el consumo del proletariado y su porcentaje respecto del consumo total, respectivamente; $\Pi$ es la ganancia que reciben los capitalistas por ser dueños de las empresas; M.S es la masa salarial; \% $\%$ es el porcentaje de las ganancias respecto del ingreso nacional; \%M.S es el porcentaje de la masa salarial respecto del ingreso nacional; Pmes, Pmec y Pmet son, respectivamente, las productividades medias del sector de los servicios, del sector convencional y total.

En el cuadro 2 se muestran los salarios y asignaciones del equilibrio restringido por la demanda. Se observa que el salario de los trabajadores que laboran en las empresas es mayor que el de los autoempleados. Sin embargo, el esfuerzo de los últimos es mayor, debido a que ellos tienen empleos más inseguros y, por tanto, se esfuerzan más para no ser despedidos, lo que motiva a sus empleadores a pagar salarios menores. Los trabajadores autoempleados representan el 14,361\% del empleo total. Adviértase que este porcentaje es menor que el que dichos trabajadores representan en la oferta de trabajo $(\phi=0,2)$, debido a que no todo el trabajo está empleado. La tasa de desempleo $(u)$ es de $5,48 \%$. La suma del consumo de bienes de los trabajadores y los capitalistas equivale al valor de la producción, porque las empresas ajustan su producción a lo que el mercado les demanda. El ingreso nacional $(I N)$ es la suma del valor de los bienes producidos por las empresas y el valor de los servicios que son proporcionados por los autoempleados $\left(S_{a}\right)$. El consumo de los capitalistas, tanto de bienes como de servicios $\left(c_{t}^{c}\right)$, representa el $57,704 \%$ del ingreso nacional, en tanto que el $42,295 \%$ restante corresponde al consumo de los proletarios. Como es de esperarse, estos porcentajes coinciden con la distribución funcional del ingreso. Finalmente, se observa que la productividad media del sector convencional $(\mathrm{Pmec})$ es superior a la productividad media del sector de los servicios ofertados por los autoempleados (Pmes); en consecuencia, la productividad media total (Pmet) es menor que la del sector convencional.

\section{Escenario 2: precarización y concentración del ingreso causadas por una mayor inseguridad en el empleo}

En este escenario se asumirá que la probabilidad subjetiva de despido de los trabajadores convencionales crece de forma sostenida, es decir, que aumenta la sensación de inseguridad en el empleo convencional. Para hacer viable esta hipótesis se parte del escenario anterior, cambiando exógenamente solo el valor del parámetro $p_{a}$. Los resultados de este ejercicio se presentan en el cuadro 3. 


\section{Cuadro 3}

Cambios en las asignaciones, los salarios y la distribución ante un incremento sostenido de la probabilidad subjetiva de despido del empleo convencional

\begin{tabular}{|c|c|c|c|c|c|c|c|c|c|c|c|c|c|}
\hline$p_{a}$ & $p_{b}$ & $w^{a}$ & $w^{b}$ & $e\left(w^{a}, p_{a}\right)$ & $e\left(w^{b}, p_{b}\right)$ & $y_{0}=\hat{y}_{d}$ & $I_{a}$ & $I_{b}$ & $S_{a}$ & IN & $\Pi$ & M.S & Pmec Pmes Pmet \\
\hline 0,02 & 0,068 & 1,943 & 1,837 & 0,515 & 0,544 & 315,163 & 61,222 & 10,685 & 19,624 & 334,787 & 196,236 & 138,550 & $5,1481,8374,656$ \\
\hline 0,03 & 0,077 & 1,918 & 1,819 & 0,521 & 0,550 & 313,677 & 60,172 & 10,899 & 19,825 & 333,502 & 198,250 & 135,252 & $5,2131,8194,693$ \\
\hline 0,04 & 0,086 & 1,895 & 1,802 & 0,528 & 0,555 & 312,300 & 59,193 & 11,105 & 20,011 & 332,311 & 200,106 & 132,205 & $5,2761,8024,727$ \\
\hline 0,05 & 0,095 & 1,874 & 1,785 & 0,534 & 0,560 & 311,010 & 58,271 & 11,305 & 20,183 & 331,193 & 201,834 & 129,359 & $5,3371,7854,760$ \\
\hline 0,06 & 0,104 & 1,853 & 1,769 & 0,540 & 0,565 & 309,793 & 57,396 & 11,499 & 20,345 & 330,139 & 203,455 & 126,684 & 5,397 1,769 4,792 \\
\hline 0,07 & 0,113 & 1,833 & 1,754 & 0,546 & 0,570 & 308,640 & 56,563 & 11,689 & 20,498 & 329,139 & 204,982 & 124,157 & $5,4571,754,4,822$ \\
\hline 0,08 & 0,122 & 1,813 & 1,738 & 0,551 & 0,575 & 307,544 & 55,766 & 11,875 & 20,643 & 328,187 & 206,427 & 121,760 & $5,5151,7384,852$ \\
\hline 0,09 & 0,131 & 1,795 & 1,723 & 0,557 & 0,580 & 306,500 & 55,002 & 12,058 & 20,780 & 327,279 & 207,797 & 119,482 & $5,5731,7234,880$ \\
\hline 0,10 & 0,14 & 1,776 & 1,709 & 0,563 & 0,585 & 305,501 & 54,268 & 12,237 & 20,910 & 326,411 & 209,101 & 117,310 & $5,6291,7094,908$ \\
\hline 0,11 & 0,149 & 1,759 & 1,695 & 0,569 & 0,590 & 304,545 & 53,562 & 12,413 & 21,034 & 325,580 & 210,343 & 115,237 & $5,6861,6954,935$ \\
\hline 0,12 & 0,158 & 1,742 & 1,681 & 0,574 & 0,595 & 303,629 & 52,881 & 12,586 & 21,153 & 324,782 & 211,528 & 113,253 & $5,7421,6814,961$ \\
\hline 0,13 & 0,167 & 1,725 & 1,667 & 0,580 & 0,600 & 302,748 & 52,224 & 12,757 & 21,266 & 324,015 & 212,662 & 111,352 & $5,7971,6674,986$ \\
\hline 0,14 & 0,176 & 1,709 & 1,654 & 0,585 & 0,605 & 301,902 & 51,589 & 12,925 & 21,375 & 323,277 & 213,748 & 109,529 & $5,8521,6545,011$ \\
\hline 0,15 & 0,185 & 1,693 & 1,641 & 0,591 & 0,610 & 301,087 & 50,974 & 13,091 & 21,479 & 322,566 & 214,788 & 107,778 & $5,9071,6415,035$ \\
\hline 0,20 & 0,23 & 1,620 & 1,579 & 0,617 & 0,633 & 297,343 & 48,156 & 13,890 & 21,935 & 319,278 & 219,353 & 99,925 & $6,1751,5795,146$ \\
\hline
\end{tabular}

Fuente: Elaboración propia.

Nota: $p, w, e(w, p)$ y / son la probabilidad subjetiva de despido, el salario real, el esfuerzo y la demanda de trabajo, respectivamente; el subíndice o superíndice "a" hace referencia al trabajador convencional y "b" al autoempleado; $y_{0}$ es la producción manufacturara; $S_{a}$ es el valor de los servicios; I $N$ es el ingreso nacional; $\Pi$ es la ganancia que reciben los capitalistas por ser dueños de las empresas; M.S es la masa salarial; Pmes, Pmec y Pmet son, respectivamente, las productividades medias del sector de los servicios, del sector convencional y total.

Como se observa en el cuadro 3 , un crecimiento sostenido de la probabilidad subjetiva de despido del empleo convencional incrementa la propia de los autoempleados, de modo que cuando $p_{a}$ es 0,02, $p_{b}$ es 0,068, pero cuando $p_{a}$ es 0,2, entonces $p_{b}$ es 0,23. Esto se debe a que al incrementarse la sensación de inseguridad en el empleo convencional, los trabajadores empleados por las empresas ven el autoempleo como un medio de evitar el desempleo, lo que tendería a incrementar la oferta de los servicios ofrecidos por los autoempleados y, por tanto, se haría más difícil la contratación para todos ellos. El incremento de la inseguridad en el empleo de ambos tipos de trabajadores los obliga a esforzarse más para evitar ser sancionados con el desempleo. En el cuadro 3 se observa que a medida que crece la probabilidad subjetiva de despido aumenta el esfuerzo que realizan los trabajadores. Sin embargo, el esfuerzo que realizan los autoempleados es siempre mayor que el que realizan los trabajadores convencionales, debido a que el empleo de los primeros es más inseguro, es decir, $p_{a}<p_{b}$ en todos los casos.

El incremento del esfuerzo de ambos tipos de trabajadores permite que sus empleadores reduzcan los salarios que pagan, debido a que ya no es necesario motivar su esfuerzo con remuneraciones altas (véanse las columnas $w^{a}$ y $w^{b}$ del cuadro 3). Sin embargo, debido a que la inseguridad en el empleo de los trabajadores convencionales crece más que la de los autoempleados, el esfuerzo de los primeros aumenta más rápido que el de los segundos y, por tanto, su salario se reduce más rápidamente que el de los autoempleados, lo que provoca una convergencia salarial a la baja, como se muestra en el gráfico 1.

La caída de los salarios de los trabajadores convencionales se traduce en una disminución de la demanda efectiva, ante lo cual las empresas contratan menos trabajo para ajustar su producción a la baja. En la columna $y_{0}=\hat{y}_{d}$ del cuadro 3 se observa la caída de la demanda efectiva y, como la producción se iguala a esta, en la siguiente columna $\left(l_{a}\right)$ se muestra la reducción del empleo que las empresas realizan para hacer coincidir su producción con la menor demanda efectiva.

En la columna $\Pi$ del cuadro 3 , se observa que la ganancia de las empresas crece debido al incremento de la diferencia entre su productividad media y el salario que pagan. 
En el gráfico 2 se visualiza cómo a medida que crece la inseguridad en el empleo, la brecha entre el índice de la productividad media y el del salario crece. El crecimiento de la productividad media de las empresas obedece al aumento del esfuerzo de los trabajadores.

\section{Gráfico 1}

Convergencia salarial a la baja

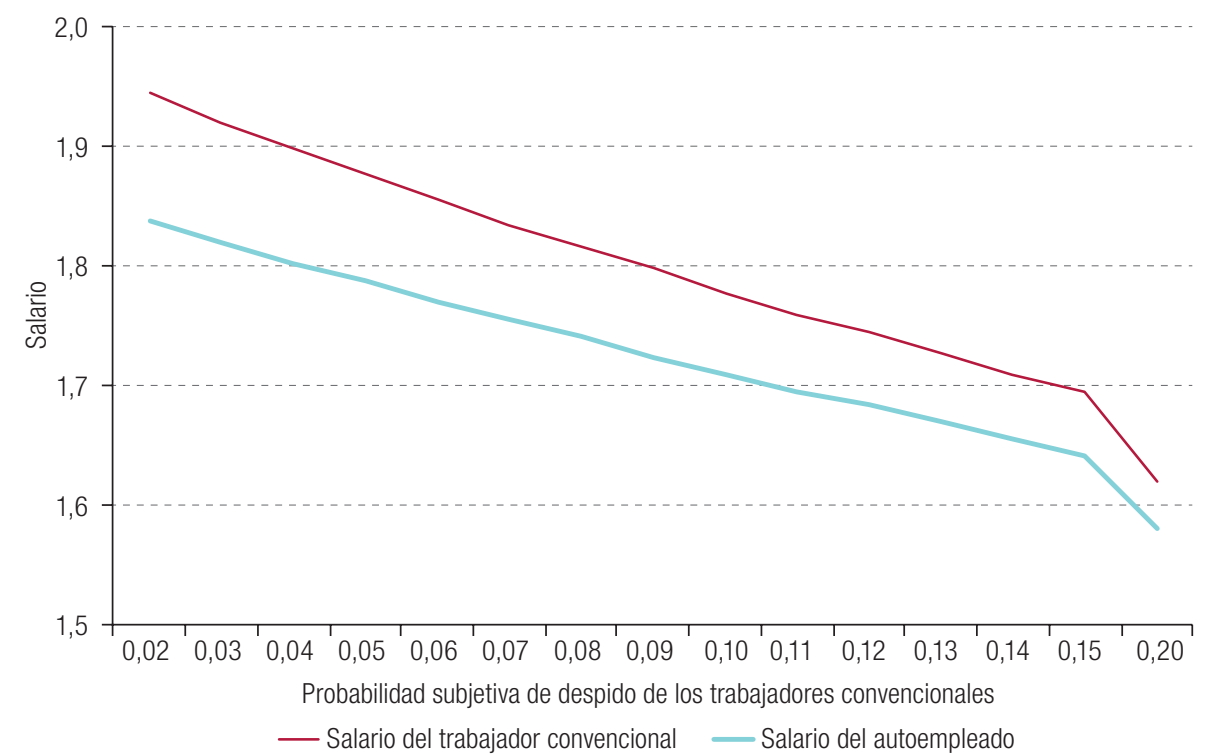

Fuente: Elaboración propia, sobre la base del salario del trabajador convencional y el salario del autoempleado, para distintos valores de la probabilidad subjetiva de despido de los trabajadores convencionales (datos presentados en el cuadro 3).

Nota: Adviértase que ambos son salarios reales, por lo que están expresados en términos de unidades de producto.

\section{Gráfico 2}

Índice de la productividad media del empleo convencional e índice del salario del trabajador convencional

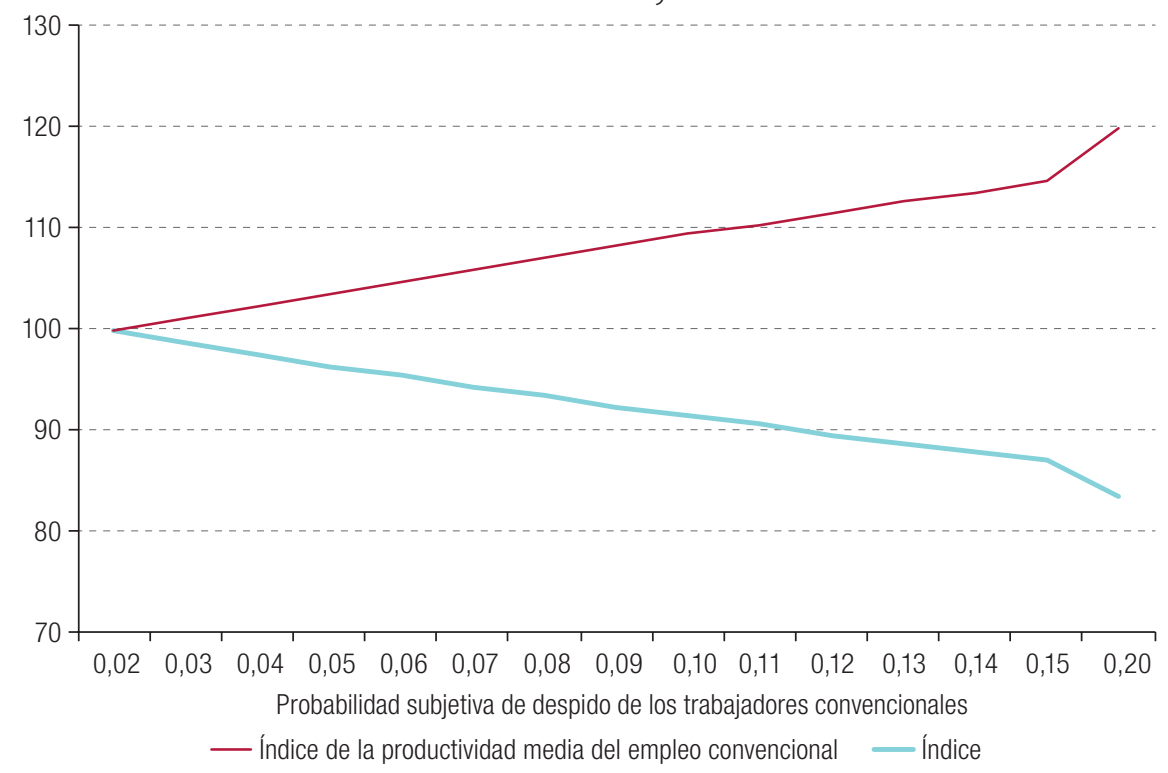

Fuente: Elaboración propia, sobre la base de los datos de productividad media del empleo convencional y de salario del trabajador convencional que se presentan en el cuadro 3. 
El crecimiento de las ganancias y la caída de los salarios de los trabajadores autoempleados provocan que los consumidores capitalistas demanden más trabajo. Como se observa en la columna $l_{b}$ del cuadro 3, el autoempleo aumenta.

El mayor autoempleo y el menor empleo convencional son coherentes con lo que evidencia la economía mexicana, en el sentido de que los empleos no precarios se han ido reduciendo y los empleos precarios han aumentado.

La reducción generalizada de los salarios, el aumento del empleo más precario y la caída del empleo convencional se traducen en que la participación de la masa salarial en el ingreso nacional se reduzca. En las columnas $\Pi$ y M.S del cuadro 3, se muestra que a medida que se incrementó la inseguridad en el empleo, la participación de los salarios en el ingreso nacional se redujo y la participación de las ganancias creció.

Finalmente, se observa que la productividad media de los servicios ofertados por los autoempleados disminuyó, debido a la caída de sus salarios, que desvaloriza los servicios que ofertan. En contraste, la productividad de las empresas aumentó, a causa del mayor esfuerzo de sus trabajadores. Ambos tipos de trabajadores incrementaron su esfuerzo; sin embargo, los efectos que esto tuvo sobre su productividad media fueron opuestos.

El gráfico 3 muestra cómo el índice de la productividad media del sector convencional crece por encima del índice de la productividad media total, debido a que el índice de la productividad media de los servicios que ofertan los autoempleados se reduce.

Gráfico 3

Índice de las productividades medias

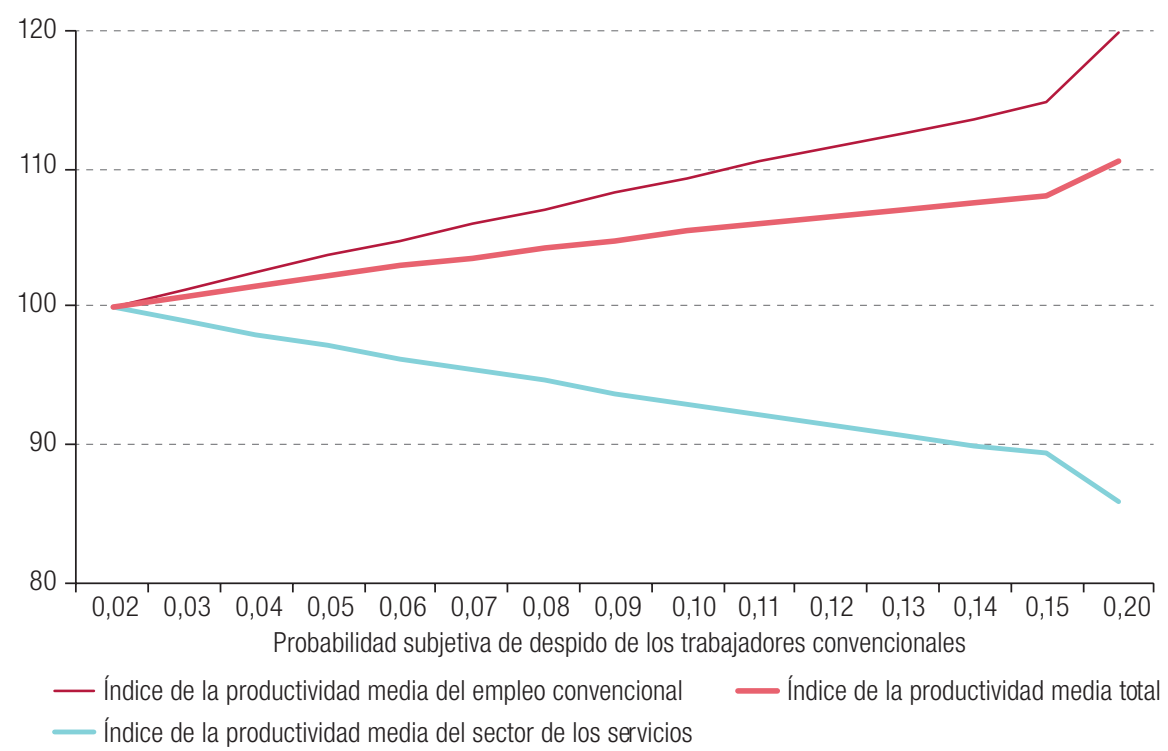

Fuente: Elaboración propia, sobre la base de los datos de las productividades medias del sector de los servicios, del sector convencional y total presentados en el cuadro 3.

Uno de los principales límites de este enfoque analítico es que no incorpora el sector externo, por lo que se trata de una economía-mundo ${ }^{13}$. Sin embargo, debido a que tanto la caída de la participación salarial como el incremento de la precariedad del empleo son dos fenómenos que se han verificado a nivel mundial, dicho modelo es válido. No obstante, puesto que la economía mexicana es una de las

\footnotetext{
${ }^{13}$ En teoría económica, cuando se modela una economía cerrada se suele argumentar que se trata de la economía-mundo, debido a que no existe una economía sin sector externo, con la excepción del mundo en su conjunto.
} 
más abiertas del mundo es necesario anotar conceptualmente cómo cambiarían los resultados en una economía abierta. El incremento de la tasa subjetiva de despido provocaría que los trabajadores se esforzaran más y, con ello, que sus salarios se redujeran. Los menores salarios en el sector exportador lo harían más competitivo, lo que fomentaría su crecimiento; por lo tanto, se tendrían escenarios de crecimiento comandado por las ganancias, siempre que el incremento de las exportaciones fuera superior a la caída del consumo de los proletarios. En este escenario podría incrementarse el empleo.

\section{Conclusiones}

El proceso de globalización productiva que se inició a finales de los años setenta y principios de los ochenta provocó un crecimiento de la inseguridad en el empleo. En este trabajo, se construyó un modelo de salarios de eficiencia, en un escenario de equilibrio restringido por la demanda, y se mostró cómo el incremento de la inseguridad en el empleo provoca que el empleo se precarice y la participación de los salarios en el ingreso se reduzca. Además de argumentarse que la precarización del empleo y la concentración del ingreso pueden tener una causa común, aunque no única, se muestra que el proceso que desencadena el crecimiento de la inseguridad en el empleo puede explicar algunas características propias de la economía mexicana.

Los resultados del modelo teórico presentado sugieren que, si se desea mejorar las condiciones de los trabajadores y revertir el proceso de concentración del ingreso, es necesario generar políticas laborales orientadas a reducir la inseguridad en el empleo.

\section{Bibliografía}

Abeles, M., V. Amarante y D. Vega (2014), "Participación del ingreso laboral en el ingreso total en América Latina, 1990-2010", Revista CEPAL, № 114 (LC/G.2629-P), Santiago, Comisión Económica para América Latina y el Caribe (CEPAL), diciembre.

Alarco, G. (2017), "Ciclos distributivos y crecimiento económico en América Latina, 1950-2014", Cuadernos de Economía, vol. 36, № 72.

_(2016), "Distribución factorial del ingreso y regímenes de crecimiento en América Latina, 1950-2012", Revista Internacional del Trabajo, vol. 135, № 1.

_ (2014), "Participación salarial y crecimiento económico en América Latina, 1950-2011", Revista CEPAL, № 113 (LC/G.2614-P), Santiago, Comisión Económica para América Latina y el Caribe (CEPAL).

Argandoña, A., C. Gámez y F. Mochón (1996), Macroeconomía avanzada, vol. II, Madrid, McGraw-Hill.

Bhaduri, A. y S. Marglin (1990), "Unemployment and the real wage: the economic basis for contesting political ideologies", Cambridge Journal of Economics, vol. 14, № 4.

Caraballo, M. Á. (1996), "Salarios, productividad y empleo: la hipótesis de los salarios de eficiencia", Cuaderno de Estudios Empresariales, vol. 6.

Clower, R. W. (1965), "The keynesian counter-revolution: a theoretical appraisal", The Theory of Interest Rate, F. H. Hahn y F. P. Brechling, Londres, Macmillan.

Colmex (2018), Desigualdades en México 2018, Ciudad de México, El Colegio de México.

Corporación Latinobarómetro (2005), Informe Latinobarómetro 2005, Santiago.

FMI (Fondo Monetario Internacional) (2007), World Economic Outlook: Spillovers and Cycles in the Global Economy, Washington, D.C.

Hein, E. (2014), Distribution and Growth after Keynes, Edward Elgar Publishing.

INEGI (Instituto Nacional de Estadística y Geografía) (2017), "Encuesta Nacional de Ocupación y Empleo (ENOE)" [en línea] https://www.inegi.org.mx/programas/enoe/15ymas/.

Kalleberg, A. L. (2009), "Precarious work, insecure workers: employment relations in transition", American Sociological Review, vol. 74, № 1.

Keynes, J. M. (1936), Teoría general de la ocupación, el interés y el dinero, Ciudad de México, Fondo de Cultura Económica. 
Luyano, C. y J. Herrera (2018), "Distribución funcional del ingreso y régimen de crecimiento en México y España", Cuadernos de Economía, vol. 37, № 73.

Noriega, F. (2001), Macroeconomía para el desarrollo: Teoría de la Inexistencia del Mercado de Trabajo, Ciudad de México, McGraw-Hill.

OIT (Organización Internacional del Trabajo) (2018), World Employment and Social Outlook: Trends 2018, Ginebra. (2011), World of Work Report 2011: Making Markets Work for Jobs, Ginebra.

OIT/OCDE (Organización Internacional del Trabajo/Organización de Cooperación y Desarrollo Económicos) (2015), "The labor share in G20 economies", Report Prepared for the G20 Employment Working Group Antaya.

Requiem for the American Dream (2015), Directed by J. P. Scott, K. Nyks, P. Hutchison, Documental, Amazon Prime Video.

Ros, J. (2015), ¿Por qué cae la participación de los salarios en el ingreso total en México?, Economía UNAM, vol. $12, \mathrm{~N}^{\circ} 36$.

(2013), Algunas tesis equivocadas sobre el estancamiento económico de México, Ciudad de México, El Colegio de México.

Samaniego, N. (2014), "La participación del trabajo en el ingreso nacional: el regreso a un tema olvidado", Economía UNAM, vol. 11, № 33.

Shapiro, C. y J. Stiglitz (1984), "Equilibrium unemployment as a worker discipline device", The American Economic Review, vol. 74, № 3.

Solow, R. (1979), "Another possible source of wage stickiness", Journal of macroeconomics, vol. 1, № 1.

Stockhammer, E. (2009), "Determinants of functional income distribution in OCDE countries", IMK Study, vol. 5.

Vázquez, D. A., I. Macias y M. Lawson (2018), "Premiar el trabajo, no la riqueza", Oxford, OXFAM International [en línea] https://www.oxfam.org/es/informes/premiar-el-trabajo-no-la-riqueza.

Velázquez, D. y Z. S. Hernández (2018), "Una explicación a la curva de salarios", Cuadernos de Economía, vol. 37, No 73.

Velázquez, D., J. R. Vargas y Z. S. Hernández (2019), "The effect of public investment on the cycle and economic growth: a simple theoretical model", International Journal of Economics and Financial Issues, vol. $9, \mathrm{~N}^{\circ} 1$. 


\section{Anexo A1}

\section{Maximización del productor}

De las primeras condiciones de máximos y mínimos se obtiene:

$$
\begin{gathered}
\frac{d(1+\pi)}{d l_{a}}=\frac{A f_{e\left(w, p_{a}\right) l_{a}}^{\prime} e\left(w, p_{a}\right) w l_{a}-w A f\left(e\left(w, p_{a}\right) l_{a}\right)}{\left(w l_{a}\right)^{2}}=0 \\
\frac{d(1+\pi)}{d w}=\frac{A f_{e}^{\prime}\left(w, p_{a}\right) l_{a} e_{w}^{\prime} w l_{a}-l_{a} A f\left(e\left(w, p_{a}\right) l_{a}\right)}{\left(w l_{a}\right)^{2}}=0
\end{gathered}
$$

De (A1) se obtiene:

$$
A f_{e}^{\prime}\left(w, p_{a}\right) l_{a} e\left(w, p_{a}\right)=\frac{A f\left(e\left(w, p_{a}\right) l_{a}\right)}{l_{a}}
$$

Adviértase que $f_{e}^{\prime}\left(w, p_{a}\right) l_{a} e\left(w, p_{a}\right)=f_{l_{a}}^{\prime}$, por lo que de (A3) se llega a la ecuación (4).

De la ecuación (A2) se obtiene:

$$
A f_{e\left(w, p_{a}\right) l_{a}}^{\prime} e_{w}^{\prime} w=\frac{A f\left(e\left(w, p_{a}\right) l_{a}\right)}{l_{a}}
$$

Sustituyendo (A3) en (A4) se obtiene la ecuación (3).

\section{Equilibrio restringido por la demanda}

Para resolver el equilibrio en el mercado de bienes se sustituyen la ecuación (21) y los respectivos planes de compra y venta en las ecuaciones (18), (19) y (20). Se obtienen:

$$
\begin{gathered}
\beta \Pi+\phi \frac{\alpha \tau^{p} w^{a}}{e\left(w^{a}, p_{a}\right)}+(1-\phi) \frac{\alpha \tau^{p} w^{b}}{e\left(w^{b}, p_{b}\right)} \gamma-A e\left(w^{a}, p_{a}\right) l_{a}=0 \\
l_{a}-\phi \frac{\alpha \tau^{p}}{e\left(w^{a}, p_{a}\right)}=0 \\
(1-\beta) \frac{\Pi}{w^{b}}-(1-\phi) \frac{\alpha \tau^{p}}{e\left(w^{b}, p_{b}\right)} \gamma=0
\end{gathered}
$$

Adviértase que los salarios se determinaron por las ecuaciones (3) y (17). Sobre la base de (A7) se obtiene que $(1-\beta) \Pi=(1-\phi) \frac{\alpha \tau^{p} w^{b}}{e\left(w^{b}, p_{b}\right)} \gamma$. Sustituyendo esta expresión, la definición de ganancia y la ecuación (A6) en el mercado de bienes (ecuación A5), se obtiene la ecuación (22), y apartir de (A7) y (A6) y la definición de se obtiene la ecuación (23). Finalmente, incorporando la hipótesis de decisión dual y sustituyendo las ecuaciones (A6) y (A7) en (21), se obtiene la ecuación (24). 


\section{Incremento de la ganancia por un aumento de la probabilidad subjetiva de desempleo}

Con la finalidad de analizar el signo de la ecuación (30), se sustituyen (29) y (27) en ella, y se obtiene:

$$
\frac{d \Pi}{d p_{a}}=l_{a-1} w_{p_{a}}^{a,}-w_{p_{a}}^{a,} l_{a}-l_{a, p_{a}}^{\prime} w^{a}
$$

Asumiendo que $l_{a-1}+l_{p_{a}}^{\prime} d p_{a} \approx l_{a}$ y sustituyendo esta idea en (A8), resulta:

$$
\frac{d \Pi}{d p_{a}}=-w_{p_{a}}^{a,} l_{a, p_{a}}^{\prime} d p_{a}-w^{a} l_{a, p_{a}}^{\prime}
$$

A partir de la ecuación (A9) se obtiene que $\frac{d \Pi}{d p_{a}}>0$, si y solo si $\left|w^{a}\right|>\left|w_{p_{a}}^{a} d p_{a}\right|$, es decir, siempre que el salario sea mayor que el valor absoluto de su reducción, lo que es cierto para todo $w>0$. 